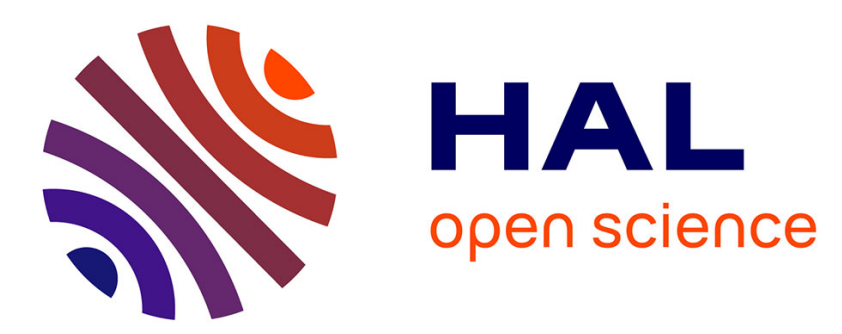

\title{
Automatic Non-Rigid Calibration of Image Registration for Real Time MR-guided HIFU ablations of mobile organs
}

Sébastien Roujol, Mario Ries, Chrit Moonen, Baudouin Denis de Senneville

\section{To cite this version:}

Sébastien Roujol, Mario Ries, Chrit Moonen, Baudouin Denis de Senneville. Automatic Non-Rigid Calibration of Image Registration for Real Time MR-guided HIFU ablations of mobile organs. IEEE Transactions on Medical Imaging, 2011, 30 (10), pp.1737-1745. 10.1109/TMI.2011.2144615 . hal01578179

\author{
HAL Id: hal-01578179 \\ https://hal.science/hal-01578179
}

Submitted on 28 Aug 2017

HAL is a multi-disciplinary open access archive for the deposit and dissemination of scientific research documents, whether they are published or not. The documents may come from teaching and research institutions in France or abroad, or from public or private research centers.
L'archive ouverte pluridisciplinaire HAL, est destinée au dépôt et à la diffusion de documents scientifiques de niveau recherche, publiés ou non, émanant des établissements d'enseignement et de recherche français ou étrangers, des laboratoires publics ou privés. 


\title{
Automatic Non-Rigid Calibration of Image Registration for Real Time MR-guided HIFU ablations of mobile organs
}

\author{
Sébastien Roujol, Mario Ries, Chrit Moonen, and Baudouin Denis de Senneville
}

\begin{abstract}
Real time magnetic resonance imaging (MRI) is rapidly gaining importance in interventional therapies. An accurate motion estimation is required for mobile targets, and can be conveniently addressed using an image registration algorithm. Since the adaptation of the control parameters of the algorithm depends on the application (targeted organ, location of the tumor, slice orientation, ...), typically an individual calibration is required. However, the assessment of the estimated motion accuracy is difficult since the real target motion is unknown.

In this paper, existing criteria based only on anatomical image similarity are demonstrated to be inadequate. A new criterion is introduced, which is based on the local magnetic field distribution. The proposed criterion was used to assess, during a preparative calibration step, the optimal configuration of an image registration algorithm derived from the Horn and Schunck method.

The accuracy of the proposed method was evaluated in a moving phantom experiment, which allows the comparison with the known motion pattern and to established criteria based on anatomical images. The usefulness of the method for the calibration of optical-flow based algorithms was also demonstrated invivo under conditions similar to thermo-ablation for the abdomen of twelve volunteers. In average over all volunteers, a resulting displacement error of $1.5 \mathrm{~mm}$ was obtained (largest observed error equal to 4-5 $\mathrm{mm}$ ) using a criterion based on anatomical image similarity. A better average accuracy of $1 \mathrm{~mm}$ was achieved using the proposed criterion (largest observed error equal to $2 \mathrm{~mm}$ ). In both kidney and liver, the proposed criterion was shown to provide motion field accuracy in the range of the best achievable.
\end{abstract}

Index Terms-Image registration, Motion analysis, Motion compensation, Magnetic resonance imaging

\section{INTRODUCTION}

$\mathbf{R}$ EAL-time motion estimation on MRI is rapidly gaining importance in interventional procedures of mobile organs (such as the heart, kidney and liver). For example, miniinvasive interventional procedures such as tissue ablation with radio-frequency (RF) and non-invasive ablation techniques such as high-intensity focused ultrasound (HIFU) show a high potential in oncology and cardiology as an alternative to classical surgery [1]. Since their objective is a necrosis

Sébastien Roujol, Mario Ries, Chrit Moonen and Baudouin Denis de Senneville* are with the Laboratory for Molecular and Functional Imaging: From Physiology to Therapy, FRE 3313 CNRS/University of Bordeaux 2, 33076 Bordeaux, France, (e-mail: \{s.roujol,m.ries,chrit.moonen,baudouin\}@imf.u-bordeaux2.fr). Asterisk indicates the corresponding author.

Sébastien Roujol is also with the LaBRI, UMR 5800 CNRS/University of Bordeaux 1, 33405 Talence, France.

Manuscript received XX XX, 2010; revised XX XX, 2010. of the tissue caused by an elevated thermal dose (TD) due to sustained heating, these methods are ideally paired with a non-invasive imaging modality which can provide both target tracking information and thermal dose measurements. The most promising candidate for this role is real-time MRthermometry based on the water proton resonance frequency (PRF) [2]. This technique provides continuous temperature mapping inside the human body as well as target tracking information by exploiting the complex nature of the MRsignal: whereas the signal magnitude $M$ provides anatomical information such as the position and the composition of the tissue, the phase $\varphi$ is directly proportional to the local proton resonance frequency and thus the local temperature. However, for moving organs the temperature information must be mapped to a reference position in order to obtain thermaldose measurements which require the voxel-by-voxel temporal integration of the temperature. An accurate estimation of organ displacement is mandatory since spatial mismatch of few millimeters may generate a perturbation of several dozen degrees [2] [3]. In addition, when the ablation is performed with a HIFU device, the estimation of the organ displacement is the basis for the dynamic adjustment of the focal point position to track the targeted pathological tissue, which prevents an inefficient treatment and unwanted destruction of healthy tissue [4].

A number of techniques have been proposed in the past to estimate on-line organ displacement, such as navigator echoes [5] or ultrasonic echoes [6]. However, the estimated motion information is generally restricted to a translational motion information. In addition, for ultrasonic echoes based approach, the estimated motion information is restricted to knowledge outside the heated zone because of the echo perturbation induced by the temperature rise. Since modern MRI acquisition methods allow the rapid acquisition of images with an excellent tissue contrast and high spatial resolution, complex deformation can be estimated on a voxel-by-voxel basis using image registration.

A variety of image registration algorithms [7] has been suggested for this purpose. Since these methods are originally developed for applications based on optical imaging, they derive displacement and deformation from anatomical (i.e. magnitude) image information [4]. However, the performance of these algorithms and the assessment of the quality of the obtained motion estimation is generally measured by criteria which are also based on the image magnitude similarity [8]. This is for MR-imaging problematic, since the fact that 
estimation and control rely on the same magnitude information renders the outcome highly dependent on the noise level of the magnitude image (which can be conveniently quantified by evaluating the signal-to-noise ratio $(S N R)$ ). Unfortunately, real-time MRI of abdominal organs is frequently hampered by low $S N R$ values of 5-20, which frequently leads to poor image registration. This problem could be alleviated if the criterion for motion estimation assessment could be based on additional information which is independent of the information used for motion estimation.

A promising way to provide this information is to exploit the phase information of the MR-image. The authors underline that it is proposed to exploit the phase component of the complex MR-signal in the image domain, not the phase associated with the fourier representation of the anatomical image [9]. While the magnitude of an MR-image reflects the underlaying anatomy, the MR-phase $\varphi$ in gradient recalled echo images is mainly determined by the local susceptibility distribution $\chi$ and the local magnetic field strength $H$ :

$$
\varphi(\vec{r}) \propto \gamma \mu_{0}(1-\sigma(T(\vec{r})))(1+\chi(\vec{r})) H(\vec{r})
$$

whereby the gyromagnetic ratio $\gamma$ and the magnetic constant $\mu_{0}$ represent material constants, $\vec{r}$ the spatial position and $\sigma$ the temperature $(T)$ dependent screening constant of the water protons. Since the susceptibility of biological tissue varies little, it is mainly the magnetic field strength $H$ which determines the spatial variation of $\varphi$. Furthermore, since modern MRsystems achieve a good homogeneity of the magnetic field strength $H$ across the field-of-view, $\varphi$ is a spatially slowly varying function which is differentiable within homogeneous tissue. These characteristics appear advantageous for a basis of tissue displacement detection.

Consequently, the objective of this study is to introduce a new criterion to quantify the quality of the estimated motion, based on the MR-phase image similarity $(P I S)$. This criterion is used to assess, for a set of positions observed before the interventional procedure, the optimal configuration of the registration algorithm. Since for HIFU ablations of mobile organs, motion is predominantly caused by the respiratory or the cardiac cycle and is thus periodic, this optimal configuration can be used during the interventional procedure. To demonstrate the usefulness of this criteria, an optical flow based image registration algorithm is optimized using both a magnitude image similarity criterion $(M I S)$ and the $P I S$ criterion for abdominal organs subjected to respiratory motion.

In general, the optimal calibration of the registration depends on several parameters (such as target motion amplitude, noise, complexity of the deformation, image resolution, ...) and thus depends on the application (targeted organ, location of the tumor, slice orientation, ...). In this paper, an image registration algorithm derived from the Horn and Schunck algorithm was optimized using our PIS criterion in a preparative calibration step.

Since HIFU ablations of mobile organs exploit the MRimage information either for the real time monitoring of the interventional process [3] or for direct retroactive control [10], the latency of the registered image stream is of great importance. For typical abdominal organ motion, the delay between the actual time of displacement and when motion information is available must not exceed $100 \mathrm{~ms}$ [10]. The implemented registration algorithm was therefore designed to be compatible with the real-time constraint and ensure short image latency. Here, the real constraint is defined as the interval of time available between two successive acquisitions, which is chosen with respect to the application.

The potential of the method was evaluated in a phantom experiment. Results of the optimal image registration were compared with gold standard positions given by an external sensor. Then, the method was demonstrated in-vivo in the abdomen of twelve volunteers under free breathing, with conditions similar to a thermo-ablation. Results of the optimal image registration were compared with manually defined gold standard positions.

\section{METHOD DESCRIPTION}

As shown in equation (1) the phase of the MR-signal represents the susceptibility distribution and the magnetic field variation in the magnet. Since both change due to organ motion, we propose to use this physical information to assess the accuracy of an estimated motion field. Two main factors, related to organ displacement, may disturb the similarity between two phase images [11]:

1) The spatial mismatch due to the displacement, which leads to a spatial mismatch of the respective magnetic susceptibility distribution $\chi(\vec{r})$.

2) Additional phase shifts generated by a modified local demagnetisation field, which is caused by a modified magnetic susceptibility distribution [11] [12] [13]. To account for these phase shifts, a precise modeling of the inhomogeneous magnetic field in-vivo is required. For that purpose, the recently suggested linear phase model approach [3] [14] assumed a simple linear magnetic field variation between the target displacement and phase variations.

Each variation of the object position leads thus to a unique phase image. If phase variations with motion have been modeled, a synthetic phase map $\left(\varphi_{\text {reco }}\right)$ can be constructed. This allows to quantify registration errors by evaluating the phase similarity between any new acquisition and the corresponding synthetic one.

A 3D image acquisition would be required. However, although it is well established that MR-imaging can provide motion estimates with a high spatial resolution, it is difficult in practice to acquire on-line 3D isotropic images because of the technical limitations, spatial and temporal resolution trade-offs, and low SNR associated with fast 3D acquisition sequences. Hopefully, in practice, it is possible for respiratory induced motion to choose the imaging plane direction parallel to the principal axis of the organ displacement and thus we evaluated the technique in two dimensions.

\section{A. Implemented image registration algorithm}

Optical flow algorithms, that was first applied to medical image registration in [15], allow estimating a velocity field 
assuming an intensity conservation during displacement, mathematically expressed by the optical flow constraint equation (OFCE):

$$
I_{x} u+I_{y} v=-I_{t}
$$

where $u$ and $v$ are displacement vector components, and $I_{x}$, $I_{y}, I_{t}$ are the spatio-temporal partial derivatives of the pixel intensity of the image.

However, a direct estimation by minimizing the deviation from the OFCE (equation (2)) is an under-determined problem and thus an additional constraint is required. The algorithm proposed by Horn and Schunck introduced an additional physical constraint by assuming that the motion field is smooth in the neighborhood of estimation point (right part in equation (3)) [16]. For that reason, this method was found to be a good candidate to estimate organ displacements during thermoablation [4]. This approach seeks a motion field minimizing:

$$
\iint\left(\left[I_{x} u+I_{y} v+I_{t}\right]^{2}+\alpha^{2}\left[\|\nabla u\|_{2}^{2}+\|\nabla v\|_{2}^{2}\right]\right) d x d y
$$

$\alpha^{2}$ is a weighting factor designed to link the two individual metrics (intensity variation and motion regularity): while low $\alpha^{2}$ values allow estimation of large motion amplitude, high $\alpha^{2}$ values increase robustness against noise or possible local intensity variations not attributed to motion. The Horn and Schunck algorithm was thus applied to anatomical (i.e. magnitude) images [16]. In order to stabilize the convergence of the algorithm, a multi-resolution scheme was used [17] which iterates the registration algorithm from a 4-fold downsampled image (where displacements are small and the $S N R$ is increased by the low-pass filtering inherent to the downsampling process) step-by-step to the full image resolution.

The optical-flow algorithm, applied on magnitude images, provides a motion field with a sub-pixel precision and an interpolation was required to obtain registered phase images. Due to the $2 \pi$ periodicity of the phase, the spatial transformation could not be directly applied on phase images. Although this problem could be circumvented by employing a 2D phase-unwrapping step to the phase images, this remains a computationally intensive processing step which is often unstable in areas with signal discontinuities and strong susceptibility changes frequently encountered in abdominal imaging. Therefore, we applied the estimated motion to the complex MR images, to obtain registered phase images, avoiding spatial phase wraps problems.

All computationally intensive calculations were offloaded to a dedicated graphics processing unit where a detailed description of the implementation can be found in [18].

\section{B. Automatic calibration of the registration}

The only required intervention of the user is to define before hyperthermia, on the reference image, a region of interest encompassing the area where the registration must be optimized (we note $m$ the associated binary mask). A reference data set was created to sample the susceptibility perturbations with motion. For most therapeutic applications, motion is caused by the respiratory or the cardiac cycle and is thus periodic. Thus, a set of $K$ images ( $K=50$ was chosen in the scope of this study) covering several motion cycles of the target with a sufficient sampling density to avoid discretization errors (5-10 images per second) was acquired. A collection of registered phase images, encoding local magnetic susceptibility variations and noted $\varphi_{r}$, was built and used to assess the quality of the registration. For that purpose, all phase images were registered to a common reference position on a pixel by pixel basis using the implemented registration algorithm applied on the anatomical (i.e. magnitude) image information. Registration errors were quantified by evaluating the phase similarity between any new acquisition $\left(\varphi_{r}\right)$ and a synthetic one $\left(\varphi_{\text {reco }}\right)$. This synthetic phase map was computed assuming a linear relation between phase variation and motion. Since the $2 \pi$ periodicity of the phase would lead to a severe bias of the similarity measure, a temporal phase unwrapping on a pixel-by-pixel basis was applied between $\varphi_{r}$ and $\varphi_{\text {reco }}$. A temporal analysis was performed to reflect the accuracy of the registration algorithm with various amplitudes and deformations likely to be encountered. For that reason, the accuracy of each estimated motion was quantified for each image of the reference dataset, and averaged to obtain the $P I S$ criterion, defined as:

$$
P I S=\frac{1}{K} \sum_{k=1}^{K}\left(\frac{1}{N} \sum_{(x, y) \in m}\left(\varphi_{r}(x, y, k)-\varphi_{\text {reco }}(x, y, k)\right)^{2}\right)
$$

Where $(x, y)$ denotes pixel coordinates, $k$ the image index in the time series, and $N$ the number of pixels set to 1 in the binary mask $m . \varphi_{\text {reco }}$ was evaluated for each individual pixel assuming a linear phase variation along the target displacement $D$ as follows:

$$
\varphi_{\text {reco }}(x, y, k)=a(x, y) \cdot D(x, y, k)+b(x, y)
$$

where $D(x, y, k)$ is a scalar relating the displacement amplitude and orientation along the principal axis of the estimated target motion computed as follow:

$$
D(x, y, k)=\Delta X(x, y, k) \cdot V_{1}+\Delta Y(x, y, k) \cdot V_{2}
$$

where $\Delta X$ and $\Delta Y$ denotes horizontal and vertical components of the estimated displacement, and $\vec{V}=\left(V_{1}, V_{2}\right)$ is the eigen vector associated to the highest eigen value.

$$
\mu=\left(\begin{array}{cc}
\cdot & \cdot \\
\cdot & \cdot \\
\overline{\Delta X}(\dot{k}-1) & \overline{\Delta Y}(k-1) \\
\overline{\Delta X}(k) & \overline{\Delta Y}(k) \\
\cdot & \cdot \\
\cdot & \cdot
\end{array}\right)
$$

where $\overline{\Delta X}(k)$ and $\overline{\Delta Y}(k)$ denote the displacement vector averaged over the mask $m$. 
$a$ and $b$ are the slope and the intercept of the simple linear regression between the registered phase value $\varphi_{\text {reg }}$ and the target displacement $D$ computed as follow:

$$
\left\{\begin{array}{c}
a(x, y)=\frac{\overline{D(x, y) \cdot \varphi_{\text {reg }}(x, y)}-\overline{D(x, y)} \cdot \overline{\varphi_{r e g}(x, y)}}{\overline{D(x, y)^{2}} \cdot \overline{D(x, y)}} \\
b(x, y)=\overline{\varphi_{\text {reg }}(x, y)}-a(x, y) \cdot \overline{D(x, y)}
\end{array}\right.
$$

The PIS criterion was used to determine the optimal $\alpha^{2}$ value in a preparative calibration step. For that purpose, an exhaustive enumeration of $\alpha^{2}$ were performed (30 $\alpha^{2}$ values were tested between 0 and 0.75 ) and the value minimizing the PIS criterion was selected as the optimal parameter.

\section{Criterion comparison}

The PIS criterion was compared with the following criteria:

a) Magnitude image similarity (MIS): The temporal average of the mean square error between each registered magnitude image $\left(M_{r}\right)$ acquired during the second step and the reference one $\left(M_{r e f}\right)$ was computed as follows:

$M I S=\frac{1}{K} \sum_{k=1}^{K}\left(\frac{1}{N} \sum_{(x, y) \in m}\left(M_{r}(x, y, k)-M_{r e f}(x, y, k)\right)^{2}\right)$

b) Gold standard error (GSE): The spatio-temporal average of the Euclidean distance between the estimated motion field and a gold standard motion information was evaluated. Since in the phantom experiment, the target undergoes a translational motion, the motion was fully characterized using a navigator echo [5]. For the in-vivo study, validation of the alignment was based on 10 landmark points, which were manually positioned and tracked over the $K$ images in the targeted region by a staff scientist with the precision of a pixel.

\section{Experimental setup}

The potential of the method to calibrate optical flow based image registration algorithms was first evaluated on a phantom experiment. Subsequently, the improved performance for the on-line estimation of organ displacements is demonstrated invivo on abdominal imaging of twelve volunteers. Dynamic MR imaging was performed on a clinical Philips Achieva 1.5 T MR-system (Philips Healthcare, Best, The Netherlands). All calculations were performed on a dual processor $(3.1 \mathrm{GHz}$ Penryn; four cores, INTEL Santa Clara, CA, USA) workstation with 8 GB of RAM. The GPU was a NVIDIA GTX280 card with $1 \mathrm{~GB}$ of DRAM. The GPU implementation was realized using CUDA.

1) Phantom study: A physiological sample with relaxation times matched to the human kidney was mounted on a motorized platform to simulate an abdominal organ moving due to respiration. The applied motion pattern consisted of a periodic sinusoidal translational displacement of $20 \mathrm{~mm}$ of amplitude with a period of $2 \mathrm{~s}$. The object displacement was monitored by an independent measure obtained by a navigator echo ( $0.5 \mathrm{~mm}$ precision), positioned parallel to the displacement, in order to get the reference displacement for the evaluation of the $G S E$ criterion. Dynamic MR imaging was performed with a dual shot gradient recalled echo-planar sequence, which employed the following parameters $(\mathrm{TR}=30 \mathrm{~ms}, \mathrm{TE}=15 \mathrm{~ms}$, voxel size $=2 \times 2 \times 5 \mathrm{~mm}^{3}, \mathrm{FOV}=256 \times 104 \times 5 \mathrm{~mm}^{3}$, echo train length $=25$, echo spacing $=1.1 \mathrm{~ms}$, flip angle $=20^{\circ}$, bandwidth in readout direction per pixel $=1777 \mathrm{~Hz}$ ). The study was investigated for different noise levels by adding an additional Gaussian noise to the initial complex data in a separate post processing step, in order to achieve series of images with an SNR of 5 to 15 .

2) In-vivo study: Dynamic MRI was performed under free breathing conditions on the kidney of 12 healthy volunteers under real-time conditions. The singleshot gradient recalled echoplanar (EPI) sequence employed the following parameters: 3000 dynamic sagittal images acquired with an imaging framerate of $10 \mathrm{images} / \mathrm{s}$, single slice, $\mathrm{TR}=100 \mathrm{~ms}, \mathrm{TE}=26 \mathrm{~ms}$, voxel size $2.3 \times 3.1 \times 6 \mathrm{~mm}^{3}, \mathrm{FOV}=300 \times 197 \times 6 \mathrm{~mm}^{3}$, echo train length $=63$, echo spacing $=0.8 \mathrm{~ms}$, flip angle $=35^{\circ}$, bandwidth in readout direction per pixel $=2085 \mathrm{~Hz}$, using a four element phased array body coil. The proposed calibration method was evaluated both in the kidney and the liver individually.

3) Statistical Analysis: The significance between the optimal $\alpha^{2}$ values obtained with the $M I S$, the PIS and the $G S E$ for a variety of noise input has been evaluated for the ex-vivo experiment using an ANOVA (Analysis of Variances) in form of a F-test with significance threshold $\mathrm{p}=0.05$. If the test was found significant, additional paired t-tests were applied to the data of all pairs of criteria. A significance of $p=0.05$ was used and corrected with the Bonferroni method.

The same statistical study was performed to assess the significance between GSE obtained with the MIS and the PIS criteria for both phantom and in-vivo experiments.

\section{RESULTS}

\section{A. Phantom study}

Magnitude and phase images obtained for two different phantom positions are displayed in Fig. 1. It can be observed that, contrary to magnitude images, phase images are not only shifted with the target displacement (shown by the red dashed lines), but also prone to an additional perturbation generated by a modified local susceptibility distribution (see red arrows in Fig. 1c and 1d).

Fig. 2 depicts the quality of the registration evaluated for each $\alpha^{2}$ value with the MIS (2a) and the PIS (2b) criteria. The GSE criterion is reported in dashed lines for comparison. Although the units are not the same, curve shapes can be compared. With both criteria it can be observed that the quality of the registration turned to be very poor for extreme $\alpha^{2}$ values (close to 0 or to 1 in this example). An optimal configuration of the implemented registration algorithm was found with both criteria: the minimum of the $M I S$ criterion was obtained for $\alpha^{2}=0.175$, and the minimum of the PIS criterion was obtained for $\alpha^{2}=0.475$. However, while the real error of the estimated displacement was 1.16 millimeters for $\alpha^{2}=0.175$, this value was 0.65 millimeters for $\alpha^{2}=0.475$.

Motion fields estimated with $\alpha^{2}=0.175$ and $\alpha^{2}=0.475$ are reported in Fig. 3c and 3d, respectively. Only the motion field 


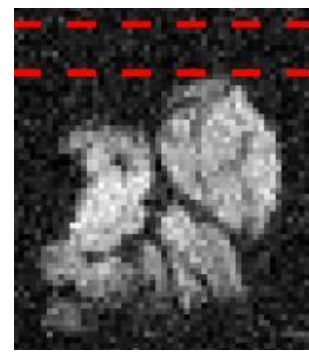

(a)

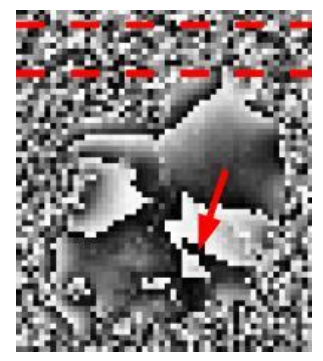

(c)

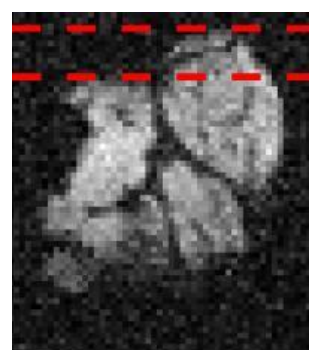

(b)

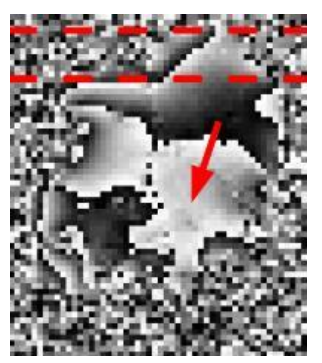

(d)
Fig. 1: Magnitude (a,b) and phase (c,d) images obtained for two different positions of the phantom with a $S N R$ of 10 . Note that the phase is a smooth function in space and the visible phase wraps are due to the $2 \pi$ periodicity of the arctan function.

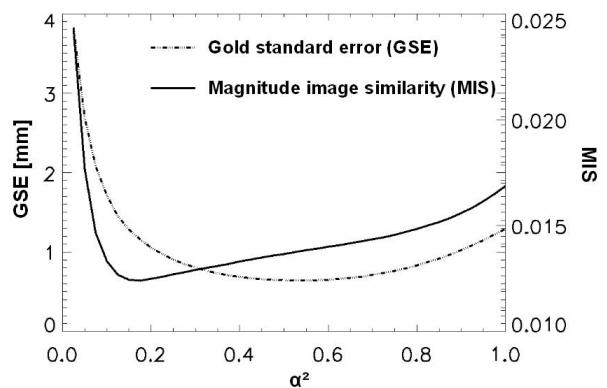

(a)

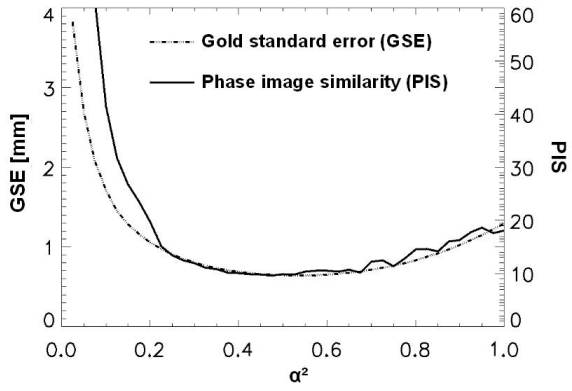

(b)

Fig. 2: Automatic determination of the $\alpha^{2}$ value for the phantom experiment ( $S N R$ was set to 10). The registration quality was assessed with the $G S E$ (dashed line) and compared to the $M S I$ (a) and the PIS criteria (b).

estimated with an optimal $\alpha^{2}$ value evaluated using our PIS criterion matched visually the real target motion measured with the transmission line (reported in Fig. 3a).

Those results were confirmed for all tested $S N R$ levels.

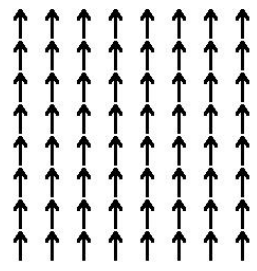

(a)

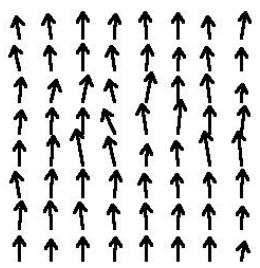

(b)

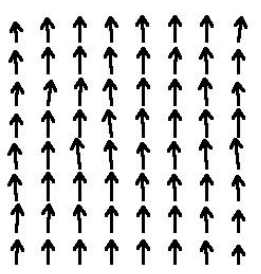

(c)
Fig. 3: (a): Gold standard motion obtained from the transmission line, (b): motion field obtained with an $\alpha^{2}$ value optimized by the $M I S$ criterion $\left(\alpha^{2}=0.175\right)$, (c): motion field obtained with an $\alpha^{2}$ value optimized by the $P I S$ criterion $\left(\alpha^{2}=0.475\right)$.

$\alpha^{2}$ values optimizing each criteria, are displayed for all tested SNR in Fig. 4a. A statistically significant difference of the optimal $\alpha^{2}$ values obtained with the MIS, the PIS and the $G S E$ for a variety of noise input could be observed $(\mathrm{p}<0.05)$. However, only the $\alpha^{2}$ optimized with the PIS didn't shown a significant difference with the $G S E$ optimization $(\mathrm{p}>0.05)$. As observed in Fig. 2, the $M I S$ criterion provided lower $\alpha^{2}$ values compared to the PIS criterion for each tested $S N R$. $\alpha^{2}$ values optimizing our criterion and the $G S E$ were similar and was found to increase from 0.4 to 0.6 with the $S N R$.

The error of the estimated displacement obtained with an $\alpha^{2}$ value optimized using the PIS, the MIS and the GSE criteria for several values of $S N R$, are reported in Fig. $4 \mathrm{~b}$. While the criteria based on anatomical image similarity led to a maximal error on the estimated displacement higher than one millimeter, the proposed PIS criterion reduced this value in the range of half a millimeter. The proposed criterion provides an accurate motion field for all tested $S N R$ levels. It can be observed that the motion field accuracy was identical when the $\alpha^{2}$ value was optimized using the PIS or the GSE criterion.

\section{B. In-vivo study}

Over the 12 human volunteers, the motion amplitude peakto-peak obtained from the landmark points was $10 \mathrm{~mm} \pm$ 4.5 (minimum=4, maximum=18) in the kidney and $11 \mathrm{~mm}$ \pm 4.5 (minimum=6, maximum=18) in the liver. The $S N R$ was evaluated to $10 \pm 2.5$ (minimum $=7$, maximum $=14$ ) in the kidney and $7 \pm 3$ (minimum=4, maximum=14) in the liver.

Fig. 5 shows a subset of the results obtained from the in-vivo experiments. Fig. $5 \mathrm{a}$ and $5 \mathrm{~b}$ show the magnitude and the phase images of the liver and the kidney in their reference position. The two manually chosen masks $m$ on the kidney and the liver (indicated in Fig. 5a by the red and blue dashed lines, respectively) were used to restrict the calibration procedure to both organs. A second set of images show magnitude and phase at a different point of the respiratory cycle (see Fig. 5c and $5 \mathrm{~d}$ ), with the contours of the reference position indicated by a dashed contour line. The maximum displacement between both images sets was in this case around $12 \mathrm{~mm}$. This second set was registered to the reference position with both the $M I S$ and the PIS calibrated registration process.

The spatial distribution of the estimated displacement amplitude is reported for the $M I S$ and the PIS criteria in Fig. 5e 


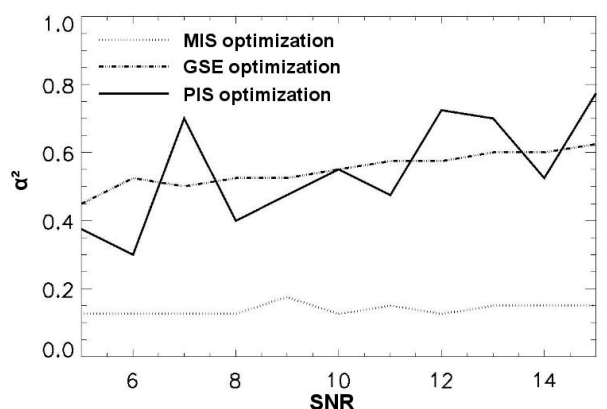

(a)

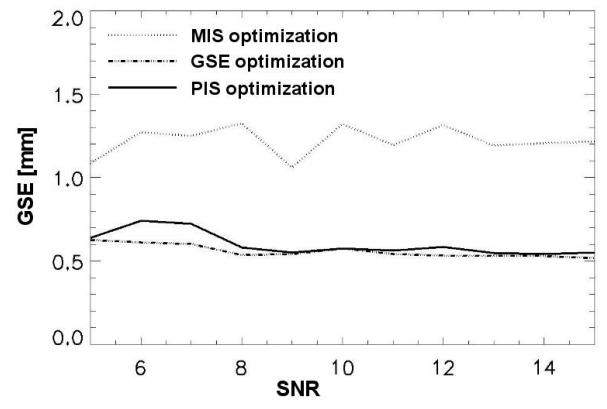

(b)

Fig. 4: (a): $\alpha^{2}$ values optimizing each criteria, for all tested $S N R$, (b): Gold standard errors obtained with optimal $\alpha^{2}$ values obtained with each criteria.

and 5f, respectively. As expected, larger displacements were observed in the upper part of the liver, close to the lung. A maximal error on the estimated displacement of 8 millimeters was measured on the landmark points with the $M I S$ in the bottom and the center of the kidney and the upper part of the liver. This maximal error decreased to $4 \mathrm{~mm}$ when the proposed $P I S$ criterion was used. Another convenient way to visualize the quality of the result is to subject masks, manually set on Fig. 5c, which depicts the anatomical contours, to the same transformation. This is shown in Fig. $5 \mathrm{~g}$ and $5 \mathrm{~h}$ respectively, where the realigned masks are overlayed as a contours over the original reference image of 5a. Note, in the two zoomed regions, the registration errors arising from the low $\alpha$ values of the $M I S$-based calibration $(\approx 8 \mathrm{~mm}$ of mismatch were observed), which are absent when the PIS was used for the calibration.

The statistical analysis over the tested volunteers of $\alpha^{2}$ values optimizing each criteria is reported for the kidney and the liver in Fig. 6.a and 6.b, respectively. Similarly to the exvivo experiment, the $M I S$ criterion provided lower $\alpha^{2}$ values compared to the PIS. The error of the estimated displacement obtained with an $\alpha^{2}$ value optimized using the PIS, the $M I S$ and the $G S E$ criteria are reported in Fig. 7.a (kidney) and Fig. 7.b (liver). For both kidney and liver, a statistically significant difference of the GSE could be observed for the $M I S$ and $P I S$ criteria in comparison to the acquisition without image registration $(\mathrm{p}<0.05)$. The $P I S$ criterion performed significantly better than the $M I S(\mathrm{p}<0.05)$, as confirmed in the Box-and-Whisker plots in Fig. 7, which represents the $G S E$ evaluation over all volunteers in both kidney (7.a) and

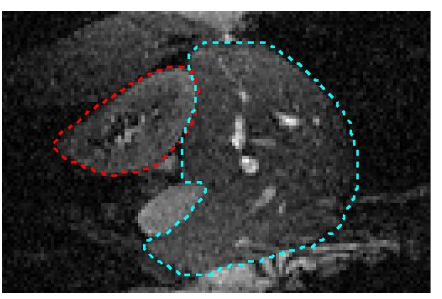

(a)

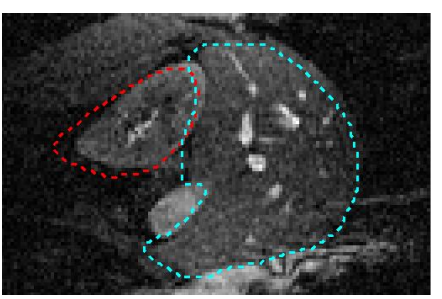

(c)

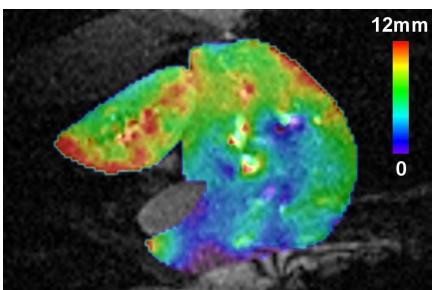

(e)

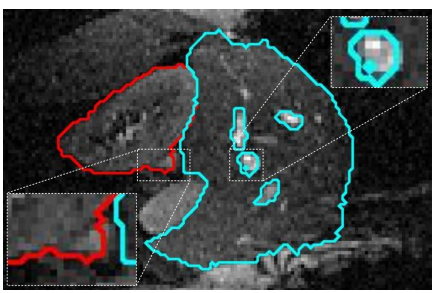

(g)

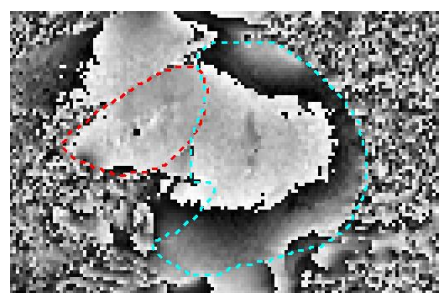

(b)

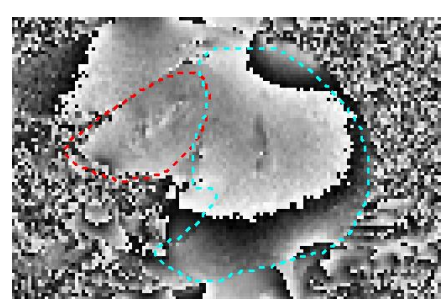

(d)

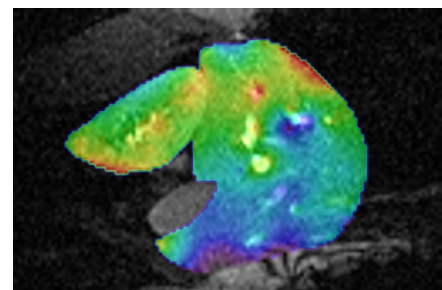

(f)

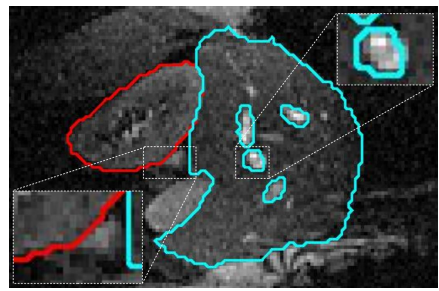

(h)
Fig. 5: Typical findings of the in-vivo study: The magnitude (a) and phase (b) image acquired at the reference position are displayed with the associated processing masks $m$ (red and blue overlays for the kidney and the liver, respectively). The corresponding shifted/deformed magnitude and phase images taken at a different point of the respiratory cycle are shown in (c) and (d). Masks $m$ at the reference position are added as dashed line to illustrate the displacement. The spatial distribution of the estimated displacement amplitude obtained with the $M I S$ and the PIS are displayed in (e) and (f), respectively. As described in the text, registration results for both calibration approaches are shown as registered masks of the shifted image overlayed to the reference image (solid lines in $(\mathrm{g})$ and $(\mathrm{h})$ ). The zoomed regions highlight the spatial mismatch between the reference image and the realigned image when a $M I S$ based calibration is used $(\mathrm{g})$, which are absent when a $P I S$ optimization is used (h).

liver (7.b). While the MIS based realignment provided a maximum displacement error of 4-5 $\mathrm{mm}$, the PIS criterion allowed to reduce this value to $2 \mathrm{~mm}$. Averaged over all volunteers, the resulting accuracy of $1.5 \mathrm{~mm}$ achieved with the $M I S$ criterion was reduced to $1 \mathrm{~mm}$ with the PIS criterion 


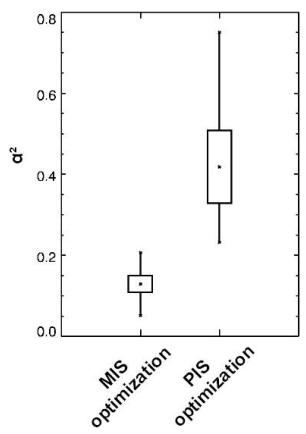

(a)

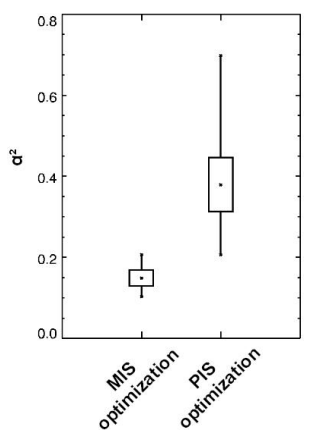

(b)
Fig. 6: Box-and-whisker plot of $\alpha^{2}$ values optimizing the $M I S$ and the PIS criteria over the 12 volunteers in the kidney (a) and the liver (b). Plotted values correspond to the minimum (lowest point), the average (cross), the maximum (highest point), and the standard deviation (box height) values across the volunteers.

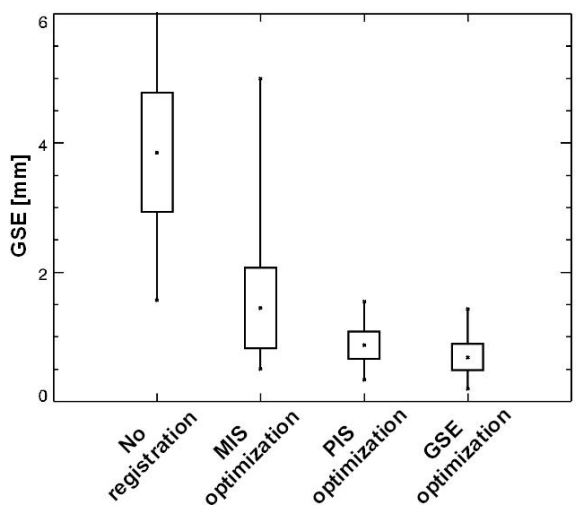

(a)

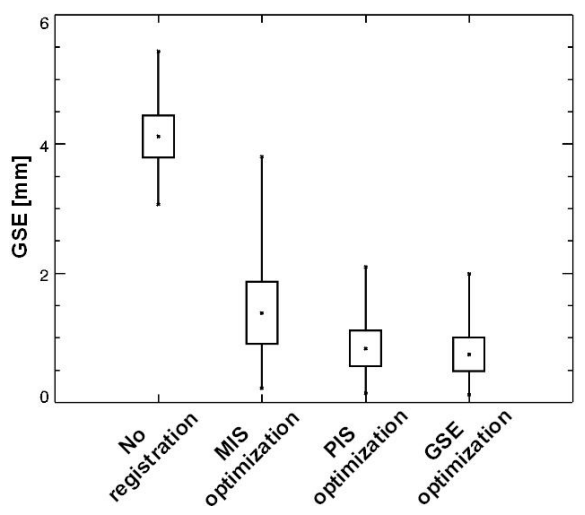

(b)

Fig. 7: Box-and-whisker plot of the gold standard errors (averaged over the $K$ images) obtained with $\alpha^{2}$ values optimizing each criteria over the 12 volunteers in the kidney (a) and the liver (b). Plotted values correspond to the minimum (lowest point), the average (cross), the maximum (highest point), and the standard deviation (box height) values across the 12 volunteers.

(which is in the range of errors similar to $G S E$ optimization).

\section{DISCUSSION}

Using the implemented Horn and Schunck algorithm, a poor estimation of the displacement is performed when $\alpha^{2}$ decreased toward 0 , due to instabilities of the numerical scheme. Identically, for $\alpha^{2}$ increasing toward infinity, tested criteria indicated a poor estimation of the displacement, as the smoothness of motion constrains the velocity amplitude estimation. The GSE criterion, as a function of $\alpha^{2}$, reflected these properties, as reported on Fig. $2 \mathrm{a}$ and $2 \mathrm{~b}$. It depicted a global minima, corresponding to an optimal registration calibration. Only the PIS matched properly this curve (see Fig. 2b).

Using the PIS criterion, the metric used for motion estimation (magnitude signal) and the metric used to evaluate the registration accuracy (phase signal) are independent. In both ex-vivo and in-vivo studies, the optimal $\alpha^{2}$ values were systematically lower using the $M I S$ criterion for the calibration compared to using the PIS criterion (see Fig. 4a and $6 a, b)$. This is explained by the fact that registration of the noise on magnitude images improved the MIS criterion. Although this may not be a limitation for video encoding in which $S N R$ is generally very high, this can be problematic in the case of fast MRI sequences which display generally a lower $S N R$, in particular for sequences providing a high temporal resolution. Replacing the MIS criterion for the calibration of the motion estimation by the PIS criterion, reduced the average displacement error by a factor of 2 for the ex-vivo experiment and 1.5 for in-vivo experiments, respectively. The difference resulting from the use of each criterion optimization was found statistically significant in all presented experiments. This demonstrates that the proposed PIS criterion outperforms the $M I S$ criterion for quantifying the accuracy of a motion estimation algorithm. In addition, the proposed PIS criterion was demonstrated to result in an optimal motion estimation similar to the best achievable estimate using the $G S E$ for all tested $S N R$ levels in the exvivo study (see Fig. 4b) and in both kidney and liver on the in-vivo study (see Fig. 7a and 7b).

Several application dependent factors require an accurate calibration of the registration. With the implemented registration model, high $\alpha^{2}$ values increased robustness against noise or possible local intensity variations not attributed to motion, but limited estimation of strong motion amplitude. In practice, the $S N R$ was found to vary a lot between volunteers due to different coil positionings. $\alpha^{2}$ value optimizing both the $P I S$ and the GSE criteria decreased for low $S N R$ values (see Fig. 4a), since less importance had to be given to grey level intensity variations in equation (3). Identically, in the invivo study, more importance was given to the displacement field regularity constraint in equation (3) for small target motion amplitudes: while $\alpha^{2}$ values higher than 0.7 were systematically found for displacement amplitudes lower than 6 mm (2 kidneys and 1 liver were concerned), $\alpha^{2}$ values lower than 0.25 were required for displacement amplitudes higher than $15 \mathrm{~mm}$ ( 2 kidneys and 3 livers were concerned).

It is interesting to observe in Fig. $2 \mathrm{~b}$ that both the PIS and the $G S E$, as a function of $\alpha^{2}$, exhibits a flat zone. A range of 
values for $\alpha^{2}$ allowing an accurate registration could thus be determined. This interval was similar for both the PIS and the GSE. This opens great perspectives to adjust freely $\alpha^{2}$ in this interval, depending on the interventional application: in the present study, we used the optimal configuration of the registration algorithm for positions observed during the calibration step. Although this should be a good solution for patient under artificial breathing or for a post-processing study, $\alpha^{2}$ may be set to the lower bound of this range for patients under free breathing, in order to allow the registration of possible larger motion amplitudes than observed during the calibration step.

The proposed PIS criterion requires an inhomogeneous magnetic field to work well, in order to obtain a spatially differentiable phase signal, which is for interventions in the abdomen for large FOVs $(>10 \mathrm{~cm})$ with current clinical equipment always the case. The implemented magnetic field perturbation model assumed a simple linear magnetic field variation with organ displacement. However, although this assumption holds in general for small displacements, the precision of this simple model showed several limitations in regions displaying large susceptibility variations, such as as in the vicinity of the digestive tube or in the upper part of the liver. Those effects explains the small difference between the PIS and GSE curves in Fig. $2 \mathrm{~b}$ and $4 \mathrm{~b}$. In addition, the linear model could be limited in the presence of through plane motion. In this case, the measured phase signal over time will not be consistent with a given tissue, and the linear relation may become inconsistent in such area. Therefore, the PIS computation may be biased in such area. However, the spatial distribution of the linear model relevance can in practice be achieved by simply mapping the fitting error $\epsilon(x, y)$ from equation (5). This also allows one to remove from the mask $m$ regions where low signal levels in conjunction with complex susceptibility variations may prevent adjustment of the linear model to the phase data.

The proposed calibration method reduced the user intervention to the determination of the mask $m$ encompassing the targeted region in the reference image. The computation time required for one image registration was $5 \mathrm{~ms}$ with the used material. The calibration process required the observation of thirty $\alpha^{2}$ values, and, for each, the registration of $K=50$ images and the PIS computation with equation (4). As an indication, less than ten seconds were required for the whole calibration process. The proposed method can thus be conveniently performed just before the hyperthermia procedure to optimize an optical-flow based registration algorithm. During the intervention, the optimized registration algorithm can be integrated in a computationally effective MR-data reconstruction pipeline [3], to provide a continuously updated target position and to feed corrections into a phased-array HIFUablator [10]. In the future, it should also be possible to combine beam steering for an effective volume ablation with the beam adaptation required for the motion compensation [19].

\section{CONCLUSION}

Although the autocalibration based on the MIS criterion resulted in a maximal error of 4-5 $\mathrm{mm}$ for the in-vivo exper- iments, the PIS criterion provided better performances that reduced this maximal error below $2 \mathrm{~mm}$. The resulting mean displacement error of $1.5 \mathrm{~mm}$ for the MIS optimization, and $1 \mathrm{~mm}$ for the PIS optimization in the in-vivo experiments were significantly different. No significant difference was observed between the estimated displacement with the proposed criterion and the real target motion in both ex-vivo and in-vivo experiments. Therefore, the proposed criterion based on MRphase images, which is based on a physical parameter (i.e. the magnetic field variation with displacement) was demonstrated to allow an automatic and efficient calibration of an image registration algorithm for real-time MR-guided HIFU ablations.

Although the proposed criterion was tested with a simple registration algorithm derived from the Horn and Schunck approach, it should be possible to extend to more complex image registration algorithms such as [20] [21]. Results obtained also open great perspectives for an evaluation of the method with different slice positioning and orientations, and other targeted organs such as in the heart.

\section{ACKNOWLEDGMENT}

The authors would like to thank the "Ligue Nationale Contre le Cancer", the "Conseil Régional d'Aquitaine", "Diagnostic Molecular Imaging” EC-FP6-project LSHB-CT-2005-512146, the "Agence Nationale de la Recherche" (project "MRgHIFUALKT") and Philips Healthcare.

\section{REFERENCES}

[1] H. Cline, J. Schenck, K. Hynynen, R. Watkins, S. Souza, and F. Jolesz, "MR-guided focused ultrasound surgery," J Comput Assist Tomogr, vol. 16, no. 6, pp. 956-965, 1992.

[2] Y. Ishihara, A. Calderon, H. Watanabe, K. Okamoto, Y. Suzuki, K. Kuroda, and Y. Suzuki, "A precise and fast temperature mapping using water proton chemical shift," Magnetic Resonance in Medicine, vol. 34, pp. 814-823, 1995.

[3] S. Roujol, M. Ries, B. Quesson, C. Moonen, and B. Denis de Senneville, "Real-time MR-thermometry and dosimetry for interventional guidance on abdominal organs," Magnetic Resonance in Medicine, vol. 63, no. 4 pp. 1080-7, 2010.

[4] B. Denis de Senneville, C. Mougenot, and C. T. W. Moonen, "Real time adaptive methods for treatment of mobile organs by MRI controlled high intensity focused ultrasound," Magnetic Resonance in Medicine, vol. 57, no. 2, pp. 319-30, 2007.

[5] J. de Zwart, F. Vimeux, J. Palussire, R. Salomir, B. Quesson, C. Delalande, and C. Moonen, "On-line correction and visualization of motion during MRI-controlled hyperthermia," Magnetic Resonance in Medicine, vol. 45 , no. 1 , pp. $128-37,2001$.

[6] M. Pernot, M. Tanter, and M. Fink, "3D real-time motion correction in high intensity focused ultrasound therapy," Ultrasound in Medicine and Therapy, vol. 30, pp. 1239-1249, 2004.

[7] J. Maintz and M. Viergever, "A survey of medical image registration," Med Image Anal, vol. 2, no. 1, pp. 1-36, 1998.

[8] D. Skerl, B. Likar, and F. Pernus, "A protocol for evaluation of similarity measures for rigid registration," Transactions on Medical Imaging, vol. 25, no. 6, pp. 779-791, 2006.

[9] D. Fleet and A. Jepson, "Computation of component image velocity from local phase information," International Journal of Computer Vision, vol. 5, pp. 77-104, 1990.

[10] M. Ries, B. Denis de Senneville, S. Roujol, Y. Berber, B. Quesson, and C. Moonen, "Real-time 3D target tracking in MRI guided focused ultrasound ablations in moving tissues," Magnetic Resonance in Medecine, vol. 64, no. 6, pp. 1704-12, 2010.

[11] J. De Poorter, C. De Wagter, Y. De Deene, C. Thomson, F. Stahlberg, and E. Achten, "The proton resonance frequency shift method compared with molecular diffusion for quantitative measurement of two dimensional time dependent temperature distribution in phantom," Journal of Magnetic Resonance Imaging, vol. 103, pp. 234-241, 1994. 
[12] S. J. Wharton and R. Bowtell, "Dipole-based filtering for improved removal of background field effects from 3D phase data," in ISMRM, 19th Annual Meeting, Stockholm, 2010.

[13] F. Schweser, B. W. Lehr, A. Deistung, and J. R. Reichenbach, "A novel approach for separation of background phase in SWI phase data utilizing the harmonic function mean value property," in ISMRM, 19th Annual Meeting, Stockholm, 2010.

[14] G. Maclair, B. Denis de Senneville, M. Ries, B. Quesson, P. Desbarats, J. Benois-Pineau, and C. T. W. Moonen, "PCA-based image registration : application to on-line MR temperature monitoring of moving tissues," in ICIP, vol. III. IEEE, 2007, pp. 141-144.

[15] J. Meunier, B. Imbert, C. Janicki, A. Guimond, and J. Soucy, "A 3-D non-linear registration algorithm for brain SPECT imaging within the Talairach reference system.” in SPIE Medical Imaging, 1997, pp. 919930.

[16] B. Horn and B. Schunck, "Determining optical flow," Artificial intelligence, vol. 17, pp. 185-203, 1981.

[17] I. Pratikakis, C. Barillot, P. Hellier, and E. Mémin, "Robust multiscale deformable registration of 3D ultrasound images," International Journal of Image and Graphics, vol. 3, no. 4, pp. 547-566, 2003.

[18] B. Denis de Senneville, K. Noe, M. Ries, M. Pedersen, C. T. W. Moonen, and T. S. Sorensen, "An optimised multi-baseline approach for on-line MR-temperature monitoring on commodity graphics hardware," in ISBI. IEEE, 2008, pp. 1513-1516.

[19] C. Mougenot, B. Quesson, B. Denis de Senneville, P. de Oliveira, S. Sprinkhuizen, J. Palussiere, N. Grenier, and C. T. W. Moonen, "Three-dimensional spatial and temporal temperature control with $\mathrm{mr}$ thermometry-guided focused ultrasound (MRgHIFU)," Magnetic Resonance in Medicine, vol. 61, pp. 603-614, 2009.

[20] H. Nagel and W. Enkelmann, "An investigation of smoothness constraints for the estimation of displacement vector fields from image sequences," Pattern Analysis and Machine Intelligence, vol. 8, pp. 565$593,1986$.

[21] N. Cornelius and T. Kanade, "Adapting optical flow to measure object motion in reflectance and X-ray image sequences," $A C M S I G$ GRAPH/SIGART Interdisciplinary Workshop on motion: representation and perception, Toronto, Canada, vol. 15, pp. 420-437, 1983. 